\title{
Ökonomie verdrängt Hippokrates - der Wertewandel im Spital
}

\author{
Brigitte Muff \\ Dr. med., Fachärztin für Chirurgie, FMH
}

\author{
Immer mehr Spitäler werden privatisiert. Flexibilität und Gewinnoptimierung \\ sind die Stichworte. Waren Chefärzte früher noch massgebende Mitglieder der Spi- \\ talleitung, übernimmt diese Aufgabe heute zur Hauptsache ein CEO. Hinzu kommt \\ die Verschärfung des Arbeitsgesetzes durch viele Zusatzbestimmungen. Können \\ Chefärzte ihren Patienten so noch gerecht werden und ihren Assistenzärzten eine \\ adäquate Weiterbildung bieten?
}

\begin{abstract}
Wenn ich nochmals wählen könnte? Ja, ich würde wieder Chirurgin werden. Für mich ist Ärztin und Chirurgin nach wie vor der Wunschberuf.

Im Team mit leitenden Ärzten, Persönlichkeiten, die alle in ihrem Fachbereich hochprofessionell arbeiten, haben wir in den letzten zehn Jahren die chirurgische Klinik des Spitals Bülach geführt. Unsere Führungskultur haben wir immer wieder überprüft und angepasst. Eine hohe professionelle Autonomie hat dies unterstützt.
\end{abstract}

Wichtig war uns eine effektive und effiziente Struktur, gepaart mit einer hohen fachlichen Qualifikation und

Economie vs Hippocrate - changement de valeurs dans les hôpitaux

Ces dix dernières années, le secteur de la santé publique a connu un changement de valeurs au profit de l'économie, non sans conséquence sur les stratégies de traitement et l'activité première des médecins. Les changements à la tête de nombreux hôpitaux ont entraîné une multiplication des fonctions administratives et managériales au détriment des activités médicales. En parallèle, l'influence et les compétences, notamment des médecins-chefs, ont fortement diminué. En outre, la détérioration de la culture de conduite par un manque de transparence et le refus d'intégrer l'expérience et le savoir-faire existants provoque la démotivation et le départ du personnel médical spécialisé et des médecins-cadres des hôpitaux publics et représente un risque pour la qualité des prestations médicales et l'efficience de I'hôpital. La transformation des hôpitaux publics en sociétés par actions et la flexibilité économique accrue visée contribuent encore à renforcer cette évolution, faisant craindre une augmentation du volume de prestations fournies et une hausse des coûts de traitement. Enfin, les exigences en partie rigides de la nouvelle loi sur le travail posent un défi supplémentaire aussi bien pour le contrôle des coûts que la qualité des prestations médicales. Un retour à la raison s'impose! einem patientenzentrierten, respektvollen und empathischen Umgang im Betrieb. Eine gute Aus- und Weiterbildung für unseren medizinischen Nachwuchs lag uns dabei besonders am Herzen.

In den letzten zehn Jahren habe ich in den schweizerischen Spitälern einen eindrücklichen Wertewandel miterlebt, der weit in die Klinikführung Auswirkungen hat. Die medizinischen Bereiche haben zugunsten der Management-Bereiche, insbesondere des Marketings oder der Unternehmensentwicklung, deutlich an Bedeutung verloren. Damit einhergehend wurde auch deren Führung, die Chefärzte, zunehmend unwichtiger und häufig aus der obersten Führungsebene verdrängt und ihre Kompetenzen eingeschränkt.

Niemand wünscht sich die Dominanz der «Götter in Weiss» zurück, doch der Einbezug der Fachkräfte und damit auch der Chefärzte in die Spitalführung ist zwingend, um die Qualität und die Effizienz der Schweizer Spitäler zu erhalten.

Diese zunehmende Ökonomisierung in der Medizin ist nicht nur bedauernswert, sondern für die medizinische Qualität unserer Spitäler auch gefährlich. Es lohnt sich deshalb, die Veränderungen genauer anzuschauen und daraus, so hoffe ich, die notwendigen Konsequenzen zu ziehen.

Die Aussagen im folgenden Artikel zu den wichtigsten Änderungen der letzten zehn Jahre resultieren in erster Linie aus meinen Erfahrungen als Chefärztin, sowie aus Diskussionen mit vielen erfahrenen Kollegen. Von Nutzen war mir dabei auch der vielfältige, vertiefte Einblick ins Schweizer Gesundheitswesen, den ich durch meine standespolitischen Aktivitäten in verschiedenen Gremien erhalten habe. 


\section{Privatisierung}

In den letzten Jahren hat eine Privatisierungswelle die Spitäler in der Schweiz erfasst. Begründet wird dies mit der Notwendigkeit, in einem sich ändernden Markt rasch reagieren zu können. Der Wettbewerb soll auch im Spital spielen und damit die gesamte Bevölkerung davon profitieren können. Die Umwandlung der Spitäler in Aktiengesellschaften führt dazu, dass monetäre Aspekte Vorrang vor dem Service-public-Auftrag erhalten haben. Nicht mehr die optimale medizinische Behandlung steht nun an erster Stelle, sondern ein

Nicht mehr die optimale medizinische Behandlung steht nun an erster Stelle, sondern ein möglicher Gewinn.

möglicher Gewinn. Dies führt dazu, dass das Leistungsangebot nicht nur genauer unter die Lupe genommen, sondern entsprechend fraktioniert wird. Die Überprüfung des Leistungsangebotes ist durchaus sinnvoll und wurde bereits früher regelmässig durchgeführt. Die Konsequenzen dieser Überprüfungen führen heutzutage jedoch zu fragwürdigen Resultaten. Einzelne Leistungsgruppen, die mehr einbringen, werden gefördert, andere, die in ihrem Gesamtvolumen z.B. zu klein sind, werden fallengelassen oder ignoriert. Das kann bedeuten, dass eine Klinik nicht mehr primär möglichst das gesamte medizinische Angebot für die Bevölkerung in einer Region aufrechterhalten will, sondern v.a. diejenigen Leistungen anbietet, die gewinnbringend sind. Gewinnbringend sind in erster Linie die Leistungen an privatversicherten Kranken.

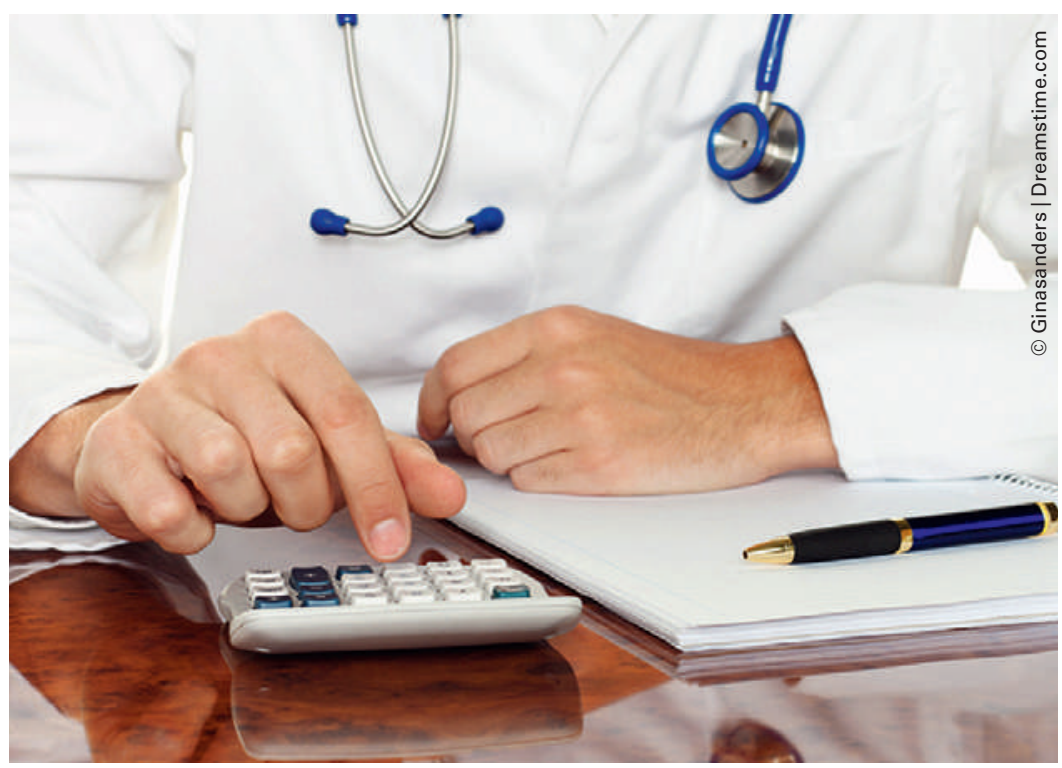

Der Taschenrechner - neben dem Stethoskop nun das wichtigste Arbeitsutensil des Arztes?
Weiter sind sie eher im stationären als im spitalspezifischen ambulanten Bereich (Handchirurgie, Schmerztherapie) angesiedelt.

Der Dienstleistungsgedanke wird zunehmend der Gewinnoptimierung geopfert. Dieses Vorgehen, früher nur das Businessmodell von Privatkliniken, hat in den letzten Jahren zunehmend die öffentlichen Spitäler erfasst.

Zusätzlich wird das erlaubte Leistungsangebot durch die «Bestimmungen der Hochspezialisierten Medizin» (HSM) von staatlicher Seite eingeschränkt, d.h. die Leistungsanbieter sind nicht frei in der Auswahl des medizinischen Angebots. Ein Widerspruch, auf den auch verschiedene Kenner des Gesundheitswesens und namhafte Ökonomen mehrfach hingewiesen haben.

So beschreibt z.B. Mathias Binswanger in seinem Buch Sinnlose Wettbewerbe (Herder) sehr anschaulich, zu welchen Absurditäten diese «Wettbewerbseuphorie» führen kann.

Zitat aus einem Interview in der TagesWoche vom März 2015: "Im Gesundheitswesen haben wir mit der Einführung der Fallkostenpauschale einen künstlichen Wettbewerb geschaffen. Dieser zwingt die Ärzte und das Pflegepersonal dazu, sich immer stärker nach ökonomischen Kriterien auszurichten. Dabei werden die Patienten zunehmend als Portfolio betrachtet, das es zu optimieren gilt.»

Über die Fallpauschalen (DRG) wurde in positiver wie auch in negativer Hinsicht bereits sehr viel geschrieben. Entscheidend ist, wie ein Abrechnungssystem eingesetzt und angewendet wird, und ob ökonomische Überlegungen die Vorherrschaft über medizinische übernehmen. Die jetzigen DRG begünstigen die Fälle mit Multimorbidität, damit bekommt der Codierer bei der Suche nach weiteren Nebendiagnosen eine entscheidende finanzielle Bedeutung.

Es ist nicht so, dass früher die Ökonomie in der Klinik keinen Einfluss hatte. Wir mussten (Neu-)Ausgaben immer begründen und hatten nie einen «Selbstbedienungsladen». Neu hat aber nicht mehr zwingend die Erfüllung des gesetzlich vorgeschriebenen Versorgungsauftrages Vorrang bei der Beurteilung der Behandlungsstrategien.

Dass die Privatisierung der Spitäler und der damit verbundene Einzug des «freien Marktes» im Gesundheitswesen nicht wirklich zu einer Kosteneindämmung führen wird, lässt sich bereits jetzt erahnen. Die Gesundheit ist ein zu kostbares Gut, um sie aufs Spiel zu setzen. Zusammen mit der Angst lässt sich dies bestens vermarkten. Eine gewisse Mengenausweitung der Leistungen hat bereits stattgefunden, doch sie ist noch lange nicht auf ihrem Zenit. Die Gesundheitskosten 
werden mit diesem teilregulierten System trotz des ökonomischen Ansatzes weiter steigen.

\section{Interne Organisation}

Unabhängig davon, ob eine Klinik privat oder öffentlich rechtlich geführt wird, sind verschiedene organisatorische Strukturen möglich. Vor zwanzig Jahren war ein sogenanntes «Dreibein» die Regel: Ärzte, Pflege und Administration führten die Klinik. Eine schlanke Führungsstruktur mit Leuten, die dem Spital oft stark verbunden, aber teilweise in organisatorischen und betriebswirtschaftlichen Fragen nicht sehr versiert waren. Dann kam die Einführung des CEO als oberste Führungsebene. Seit gut zehn Jahren ist dies praktisch durchgängig der Fall. Dies führte zu weiteren Änderungen in der Spitalstruktur, z.T. wurde dabei die Spitalleitung wie in Bülach auf sehr viele Personen erweitert (bis 15 Bereichsleitungen).

\section{In den letzten Jahren wurde der Einfluss der Ärzteschaft in vielen Spitalleitungen zunehmend abgebaut.}

Als ich vor zehn Jahren den Chefarztposten Chirurgie übernahm, war eine Beteiligung einer starken Ärzteschaft in der Geschäftsleitung noch unbestritten und meist auch erwünscht. In den letzten Jahren wurde der Einfluss der Ärzteschaft in vielen Spitalleitungen aber zunehmend abgebaut. Es gibt Kliniken, bei denen man das Gefühl hat, dass die Chefärzte nur noch am Rande oder als "Alibi-Bereichsleiter» in der Geschäftsleitung vertreten sind, d.h. es fehlt ihnen die Zeit, um sich neben der klinischen Tätigkeit genügend mit den Geschäftsleitungsthemen oder den zusätzlichen Führungsfunktionen auseinanderzusetzen oder sie sind kaum mehr fachlich tätig, was zu mangelnder «Praxisnähe» und Akzeptanz führen kann.

Die faktische oder formale Abwesenheit der Fachkräfte in der obersten Führungsebene führt zu mangelnder Transparenz, ungenügender Kommunikation und damit zu einem Vertrauensverlust in die Führung. Die Balance zwischen Verantwortung und Mitbestimmung bei den Chefärzten wird empfindlich gestört. Obwohl von einem Anwärter auf einen Chefarztposten auch heute noch eine betriebswirtschaftliche Weiterbildung verlangt wird, kann er in den neuen Strukturen dieses Wissen nicht mehr adäquat einsetzen. Bei wichtigen Entscheiden auf der Verwaltungsratsebene wird er nicht mehr miteinbezogen. Dies führt zu einem Motivations- und Initiativverlust.

Dafür werden Kaderleute auf der operativen Ebene vermehrt in Projekte und administrative Aufgaben ein- gebunden. Es wird strukturiert und in regelmässigen Abständen restrukturiert, meist unter Beizug von teuren externen Beratern ohne spezifisches Know-how im Gesundheitsbereich. Es ist manchmal schwierig den Überblick zu behalten und jederzeit zu wissen, was bezüglich ablauforganisatorischer Regelungen gerade gilt. Zunehmend werden Chefärzte auch bei Anschaffungen übergangen, die ihre Arbeit direkt betreffen. Auch ein Wandel in der Kommunikation hin zu juristischen oder pseudojuristischen Disputen ist zu beobachten.

Ich habe in den zehn Jahren Chefarzttätigkeit den mehr oder weniger freiwilligen, jedoch immer vorzeitigen Abgang von fünf Chefärzten und mindestens ebenso vielen Leitenden Ärzten oder Chefarztstellvertretern miterlebt. Der Weggang erfolgte meistens wegen «Unstimmigkeiten» mit der Geschäftsleitung. Nur einer davon geschah aus anderen persönlichen Gründen. Jedes Mal war dies mit grosser Unruhe und entsprechenden Einbussen bei den Behandlungszahlen und den Zuweisungen verbunden. Meistens folgten dann Monate dauernde, unbefriedigende Übergangslösungen, verbunden mit zusätzlicher Verunsicherung der Mitarbeitenden. Situationen, die für einen kontinuierlichen, qualitativ hochstehenden medizinischen Betrieb kaum förderlich sind.

Die Änderung in der Organisationsstruktur, die die Abschaffung der Vertretung genügender Fachkompetenz in den Führungsgremien zur Folge hat und die Chefärzte zu reinen "Befehlsempfängern» degradiert, ist vermutlich der häufigste und schwerwiegendste Grund, weshalb Chefärzte von einer Klinikführung Abstand nehmen oder diese vorzeitig verlassen.

\section{Auswirkungen des Arbeitsgesetzes}

Bereits vor zehn Jahren war die Beschränkung der Arbeitszeit für Assistenzärzte auf 52 Stunden pro Woche eingeführt. Ohne weitere Einschränkungen konnten Überstunden gesammelt und dann als mehrwöchige Ferien/Kompensation oder einzelne Freitage bezogen werden. Die kontinuierliche Betreuung der Patienten konnte so gewährleistet werden. Die überlangen Dienste von 36 oder gar 48 Stunden, die auch in Medizinerkreisen zunehmend als verantwortungslos angesehen wurden, fielen weg. Niemand vermisste die durchschnittlichen Arbeitszeiten von 80 Stunden und mehr pro Woche. Die Lebensqualität wurde damit im Vergleich zu früher deutlich besser. In den letzten zehn Jahren ist das Arbeitsgesetz aber in einer Weise verschärft worden, in der eine kontinuierliche Betreuung der Patienten durch die gleichen Verantwortlichen nicht mehr gewährleistet werden kann. Häufige Wech- 
sel in der Betreuungscrew sind als Risiko für die Behandlungssicherheit bekannt, da unter anderem bei den vielen Übergaben Informationen verloren gehen. Der Unmut über diese Situation zeigt sich auch in den Patientenumfragen, in denen der häufige Wechsel der zuständigen Medizinalpersonen (Ärzte und Pflegepersonen) als einer der meistgenannten negativen Punkte aufgeführt wird.

Auch wenn alle froh sind (auch die Chefärztinnen), dass die unsinnig langen Arbeitszeiten abgeschafft worden sind, stellt sich die Frage, ob das in einem adäquaten Rahmen passiert ist. Ich kenne niemanden, der eine 50-Stunden-Woche verurteilen würde. Im Rahmen einer entsprechenden Ganzjahresarbeitszeit ist dies kein Problem. Es ist die «Zerstückelung» der Arbeitszeit durch viele zusätzliche Bestimmungen, die eine vernünftige, kontinuierliche Patientenbetreuung verhindert. Es ist nicht die absolute Zahl von 50 Stunden pro Woche, sondern die vielen «Wenn-Dann», welche vernünftige Dienstpläne verunmöglichen. Auch Überstunden dürfen nur noch in begrenztem Umfang gemacht werden, da eine kumulierte jährliche Obergrenze von maximal 140 Überstunden festgesetzt ist.

Eine adäquate Weiterbildung der Assistenzärzte (und teilweise auch der Oberärzte) wird mit diesen einschränkenden Bestimmungen stark erschwert und ist mit negativen Konsequenzen verbunden. Die zur Ausübung unseres Berufes notwendige Erfahrung, die vor allem auch durch eine kontinuierliche, z.T. zeitintensive Behandlung und Betreuung der Patienten gesammelt werden kann, wird wegen der einschränkenden Verordnung kaum mehr erreicht.

Es ist wichtig, dass die maximale Arbeitszeit eingehalten wird, da sind sich mittlerweile alle Betroffenen einig. Es ist aber aus Patientensicht ebenso wichtig, dass die rigiden Arbeitszeitbeschränkungen gelockert werden, damit die Sicherheit und das Wohl der Patienten durch Kontinuität der Betreuung und intensive Weiterbildung der Fachärzte verbessert werden.

\section{Persönliches Fazit}

Korrespondenz:

Dr. med. Brigitte Muff

Fachärztin für Chirurgie

Möhrlistrasse 124

CH-8006 Zürich

bmuff[at]bluewin.ch unterhalt mit Tätigkeiten im Gesundheitswesen. Zwar hat die Anzahl der Ärzte wegen des strengeren Arbeitsgesetzes in den letzten Jahren ebenfalls zugenommen, jedoch nicht im gleichen Ausmass wie beim Personal, das keinen direkten Patientenkontakt hat. Die prozentual grösste Zunahme dürfte in den letzten zehn Jahren im Marketing- und im Managementbereich stattgefunden haben.

Parallel dazu hat auch der beschriebene Wertewandel vom Primat der Medizin hin zur Priorisierung der Ökonomie stattgefunden und damit verbunden ein Verlust von Einfluss von Fachkräften in der Klinikführung.

Immer mehr Leute verdienen ihren Lebensunterhalt mit Tätigkeiten im Gesundheitswesen.

Die Auswirkung dieser Entwicklung auf die Kultur in den Kliniken und damit auch auf die Qualität der medizinischen Verrichtungen darf nicht unterschätzt werden. Anstelle der Motivation durch vorbildhafte Leistung und Kompetenz des medizinischen Kaders und guten Teamgeists sollen heute Budgetvorgaben, Weisungen, Richtlinien und Sanktionen Leistung und Qualität bewirken. Die Folge davon ist Demotivation und Frustration auf allen Ebenen des medizinischen Personals, vor allem aber bei engagierten Kaderärzten. Das medizinische Leitungsteam einer Klinik muss zwingend seine eigene Kultur prägen können und diese natürlich immer wieder hinterfragen. Aber auch die Gesamtkultur eines Spitals muss immer wieder hinterfragt und zusammen mit dem ärztlichen Kader gestaltet werden. Dies findet zunehmend weniger statt und führt u.a. dazu, dass ärztliche Führungsstellen im Spital uninteressant werden.

Ein Umdenken ist aus all diesen Gründen dringend nötig.

Wer sich vertieft mit der Ökonomisierung in der Medizin befassen möchte, dem empfehle ich die Lektüre der Bücher von Bernhard Lown: Die verlorene Kunst des Heilens (Schattauer) oder des Medizinethikers Giovanni Maio: Geschäftsmodell Gesundheit: Wie der Markt die Heilkunst abschafft (Suhrkamp).

\section{Danksagung}

Ich bedanke mich im Besonderen bei meiner Freundin Ursula Kündig, sowie bei zwei nicht genannt sein wollenden Kollegen für das kritische Redigieren des Textes, sowie bei Dr. med. H.-U. Würsten (Präsident VLSS) und Dr. iur. Th. Eichenberger (Geschäftsführer VLSS) für die Durchsicht des Artikels. 